\title{
CARACTERIZACIÓN FISICOQUÍMICA Y EVALUACIÓN DE LA ACTIVIDAD ANTIOXIDANTE DEL ACEITE DE Euterpe precatoria Mart. OBTENIDO POR DIFERENTES MÉTODOS DE EXTRACCIÓN
}

\author{
Ortega Romero Elizabeth ${ }^{\mathrm{a}^{*}}$, Jurado Teixeira Bertha ${ }^{\mathrm{b}}$, Ramos Llica, Eva ${ }^{\mathrm{b}}$, Zamudio \\ Malpatida Karin', Aparicio Aquise Elvira ${ }^{b}$.
}

\begin{abstract}
RESUMEN
Se investigó los frutos de Euterpe precatoria Mart. (asaí) de Yurimaguas del Departamento de Loreto, según sus caracteres morfológicos y análisis proximal; se obtuvo aceite a través diferentes métodos, evaluándose el rendimiento de extracción, propiedades fisicoquímicas y actividad antioxidante. Los resultados morfológicos indican longitudes, diámetros y peso de frutos iguales a $1,324 \mathrm{~cm} 1,425 \mathrm{~cm}$ y $1,797 \mathrm{~g}$, respectivamente. El análisis químico proximal se realizó a la pulpa seca, la cual fue utilizada para la extracción del aceite. El contenido de cenizas, fue similar a lo reportado para la especie de Euterpe olaraceae Mart. El contenido de lípidos totales fue superior a lo descrito de un contenido de lípidos de $11,5 \%$ en pulpa sin tratamiento, pero menor a lo reportado (37\%) en otros estudios. El rendimiento por prensado y pre tratamiento enzimático-prensado fue 31,9\% y 35,3\%, respectivamente. Sin embargo, no se determinó una diferencia de rendimiento estadísticamente significativa al compararlos. El rendimiento por extracción con solventes fue 42,6\% y se observó diferencias en la intensidad de color y el IC 50. El IC 50 del aceite obtenido por prensado y pre tratamiento enzimático fue $6,97 \mathrm{mg} / \mathrm{mL}$ y $3,61 \% \mathrm{mg} / \mathrm{mL}$, respectivamente; el mejor resultado fue la extracción por solventes IC $501,82 \mathrm{mg} / \mathrm{mL}$; sin embargo, ninguno de los extractos mostró mayor actividad que el Trolox (IC 50 0,001 mg/mL). El contenido de fenoles totales de los aceites obtenidos fue superior a los resultados reportados para E. oleracea, observándose una mayor presencia de compuestos fenólicos en la especie E. precatoria. Confirmando lo reportado respecto al predominio de compuestos fenólicos en E. precatoria, en comparación a E. oleraceae.
\end{abstract}

Palabras clave: Asaí, Euterpe precatoria Mart., extracción de aceites, capacidad antioxidante.

\section{PHYSICOCHEMICAL CHARACTERIZATION AND EVALUATION OF ANTIOXIDANT ACTIVITY OF OIL FROM Euterpe precatoria Mart. OBTAINED BY DIFFERENT METHODS OF EXTRACTION}

\section{ABSTRACT}

The fruits of Euterpe precatoria Mart.(acai) were investigated from Yurimaguas-Loreto

\footnotetext{
a* Facultad de Farmacia y Bioquímica, Universidad Nacional Mayor de San Marcos, Jr. Puno 1002, Lima, Perú, elizabeth.ortega16@gmail.com

b Instituto de Investigación en Recursos Naturales y Terapéuticos "Juan de Dios Guevara" Facultad de Farmacia y

Bioquímica, Universidad Nacional Mayor de San Marcos, Jr. Puno 1002, Lima, Perú.

c Amazon Andes Export S.A.C, Cal. Ruy Diaz Nro. 239, Lima, Perú
} 
Department, according to their morphological and proximate analysis; oil was obtained through different methods, evaluating the extraction yield, physicochemical properties and antioxidant activity. The morphological results indicate lengths, diameters and fruit weight equalto $1,324 \mathrm{~cm}$ and $1,425 \mathrm{~cm}$ and $1,797 \mathrm{~g}$, respectively. The proximate analysis was performed to dry pulp, which was used to extract the oil. The ash content was similar to that reported for the species Euterpe olaraceae Mart. The total lipid content was higher than that described a lipid content of $11,5 \%$ in untreated pulp, but lower than that reported $(37 \%)$ in other studies. Yield by enzymatic pre-pressing and pressing treatment was 31,9\% and 35,3\% respectively. However, a statistically significant difference when compared yield was not determined. The solvent extraction yield was $42,6 \%$ and was observed differences in color intensity and IC50. The IC50 of the oil obtained by pressing and enzymatic pretreatment was $6,97 \mathrm{mg} / \mathrm{mL}$ and $3,61 \mathrm{mg} / \mathrm{mL}$, respectively; the best result was solvent extraction IC50 1,82 mg/ $\mathrm{mL}$; however, none of the extracts showed greater activity than Trolox(IC 500,001 mg/ mL). The total phenolic content of oils obtained was higher than the results reported E.oleracea Mart, with a greater presence of phenolic compounds in the species E.precatoria. Confirming reported to prevalence of phenolic compounds in E.precatoria compared to E.oleraceae.

Key words: Acai, Euterpe precatoria Mart., extraction of oils, antioxidant capacity.

\section{INTRODUCCIÓN}

Euterpe precatoria Mart. es una palmera nativa de Sudamérica, localizada principalmente en la Amazonía peruana, boliviana y brasileña, conocida comúnmente como palmito, manaca, chonta, asaí de la selva o huasaí1-4. Su consumo data de tiempos precolombinos y es un alimento muy importante en la dieta amazónica ya que sus frutos y derivados son altamente oleaginosos y de sabor agradable ${ }^{5-10}$. Bajo el nombre común de asaí se comercializa dos especies diferentes: Euterpe oleracea Mart. y Euterpe precatoria Mart. Se diferencian entre sí a nivel morfológico y en la composición fitoquímica, reflejada en la mayor capacidad antioxidante de E. precatoria. ${ }^{11}$ En relación al perfil de ácidos grasos, Euterpe oleracea que reporta alta cantidad de ácidos grasos poliinsaturados ( $71 \%$ del total de lípidos), destacándose el alto contenido de ácido oleico (18:1), seguido de linoleico (18:2) y $\alpha$-linolénico (18:3), siendo los valores en ácido oleico comparables a lo aportado por el aceite de oliva y canola $(77,0 \text { y } 61,5 \% \text {, respectivamente })^{12-14}$. Este aceite reconocido como un producto exótico cuyos componentes están asociados al efecto antienvejecimiento, antioxidante y regenerador de la piel; de ahí su aplicación cosmética y dermatológica. El objetivo del presente trabajo fue evaluar el rendimiento en aceite a través de diferentes métodos de extracción, caracterizar el aceite a nivel fisicoquímico y evaluar la actividad antioxidante.

\section{Material biológico}

\section{PARTE EXPERIMENTAL}

Los frutos de Euterpe precatoria Mart. utilizados en la investigación fueron recolectados en la comunidad San Francisco - río Amazonas y comunidades aledañas a la altura de la carretera Iquitos- Nauta (kilómetros 10 al 60), en la provincia de Maynas, Loreto - Perú. Todos los reactivos y solventes utilizados fueron de grado analítico de las marcas Sigma Aldrich Chemical Co. y Merck. 


\section{Métodos}

Evaluación morfológica de los frutos: Muestras representativas de 30 frutos de un lote de producción fueron utilizados para medir la longitud de fruto desde la base hasta el ápice $(\mathrm{cm})$, diámetro de fruto en la parte central $(\mathrm{cm})$, peso de fruto $(\mathrm{g})$, peso de cáscara $(\mathrm{g})$ y peso de pulpa (g).

Análisis químico proximal de pulpa: Humedad (AOAC 925.40 - Moisture in Nuts and Nut Products), grasa (AOAC 948,22 Fat (crude) in nuts and nut products.), cenizas (AOAC 950,49-Ash of Nuts and Nut Products), fibra cruda (AOAC 935,53- Fiber (Crude) in Nuts and Nut Products), proteínas (AOAC 950,48-Protein (crude) in nuts and nut products) y carbohidratos (por diferencia); fueron determinados por métodos oficiales de la $\mathrm{AOAC}^{15}$.

Análisis del aceite crudo: Índice de acidez $(940,28)$, índice de yodo $(920,159)$, índice de peróxido $(965,33)$, índice de refracción $(921,08)$ y densidad $(920,212)$; fueron determinados por métodos oficiales de la $\mathrm{AOAC}^{15}$.

Análisis de la actividad antioxidante: A partir de cada extracto de aceite; se pesó 1 gramo y se agregó $3 \mathrm{~mL}$ de mezcla solvente (metanol: agua (80:20) v/v) (Parry et al., 2005) ${ }^{16}$; se agitó en vortex y centrifugó a $35000 \mathrm{rpm}$ por $15 \mathrm{~min}$; el sobrenadante fue colectado; la extracción se realizó por triplicado y los tres sobrenadantes fueron mezclados. El volumen final fue completado a $10 \mathrm{~mL}$ con la mezcla solvente y fueron conservados en refrigeración y protegidos de la luz.

Para la evaluación de la inhibición de radicales libres se empleó el método DPPH (1,1-difenil2-picril-hidrazilo) modificado por Brand William et al., $1995^{17}$. En la evaluación de fenoles totales se aplicó el método de Folin-Ciocalteu, modificado por Chaovanalikit y Wrolstad $(2004)^{18}$. Se tomó $0,1 \mathrm{~mL}$ del extracto del aceite, fue mezclado con 0,5 mL de $2 \mathrm{~N}$ FolinCiocalteau y 7,9 mL de agua desionizada. La mezcla se conservó a temperatura ambiente por $10 \mathrm{~min}$. Luego se agregó $1,5 \mathrm{~mL}$ de una solución de carbonato de sodio al $20 \%$. La mezcla fue llevada a reposo por 2 horas y la absorbancia fue leída a $765 \mathrm{~nm}$. La cuantificación de fenólicos totales fue realizada usando una curva de calibración de ácido gálico (100-2000 ppm) y los resultados fueron expresados como equivalentes de ácido gálico (EAG) mg/100g de aceite.

Análisis estadístico: Todos los resultados fueron analizados en el software estadístico IBM SPSS Statistics22. Para la comparación de la eficiencia de métodos de extracción se aplicó la prueba de homogeneidad de varianzas (estadístico de Levene) y se realizaron comparaciones múltiples entre los métodos de extracción con las pruebas T3 de Dunnett, C de Dunnett, Scheffé y Games-Howell. Para los resultados de análisis físico químico y actividad antioxidante de los aceites obtenidos a través de los métodos de extracción en estudio, se aplicó la prueba de homogeneidad de varianzas (estadístico de Levene) y comparaciones múltiples con las pruebas HSD de Tukey y el test de Scheffé.

\section{Procedimiento experimental para la extracción del aceite}

Para el prensado en frío se utilizó una prensa hidráulica. Se pesó de 500 a 650 g de pulpa (humedad 12\%). Para la digestión enzimática se utilizó el complejo enzimático Pectinex ${ }^{\circledR}$ Ultra SPL - Sigma Aldrich (solución acuosa de pectinasa de Aspergillus aculeatus, $\geq 3,800$ units $/ \mathrm{mL}$ ); se realizó en marmita con agitador en hélice de acero inoxidable; la pulpa digestada se secó hasta $12 \%$ de humedad y se prensó. En la extracción por solventes se 
utilizó el equipo de la planta piloto de extracción sólido-líquido (C) ElettronicaVeneta S.p.A. del Laboratorio de Operaciones Unitarias de la Universidad Nacional del Callao. El aceite obtenido fue calculado gravimétricamente. Los parámetros de extracción, de cada método en estudio, se muestran en la figura 1.

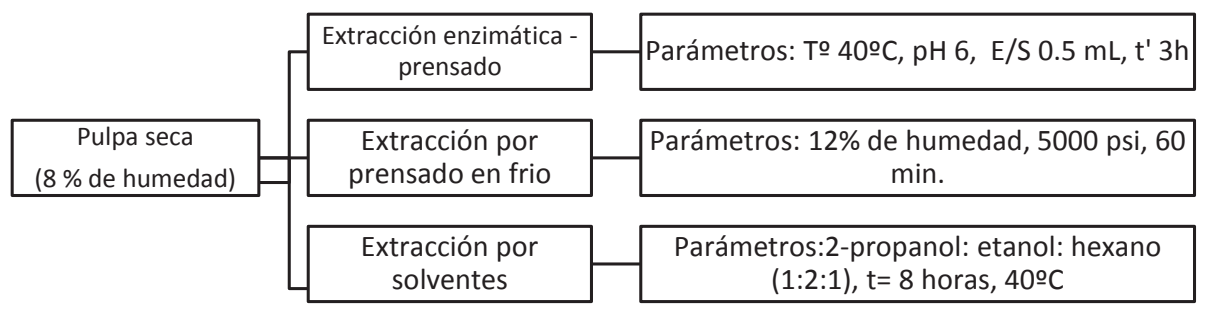

Figura 1. Métodos de extracción y parámetros aplicados en la obtención del aceite de Euterpe precatoria Mart

\section{RESULTADOS Y DISCUSIÓN}

En la tabla 1 se muestra las características morfológicas del fruto de Euterpe precatoria Mart. gran parte del peso del fruto es semilla; el exocarpo y mesocarpo que envuelven la semilla representa sólo el 23\% del peso aproximadamente. Se evaluó 30 frutos de Euterpe precatoria Mart.; el color de la cáscara del fruto es púrpura oscuro, interiormente la coloración varía de verde a naranja oscuro. El exocarpo es muy fino, de color púrpura violeta y el mesocarpo posee sólo 1 a $2 \mathrm{~mm}$ de espesor; el exocarpo y mesocarpo constituyen la parte comestible de la fruta, denominada "pulpa". La semilla de Euterpe precatoria constituye el $83 \%$ de la fruta y está formado por un pequeño endospermo sólido, unido a una cubierta de la semilla en la madurez; estos resultados se aproximan mucho a lo descrito por Barriera y Rogez ${ }^{17}$, y el Serviço Florestal Brasileño ${ }^{4}$, los que señalan un peso promedio del fruto de 0,8 a 2,3 g, 17\% de pulpa y diámetro de 1-2 cm (figura 2).

Tabla 1. Evaluación morfológica de los frutos de Euterpe precatoria*

\begin{tabular}{lc}
\hline Longitud $(\mathrm{cm})$ & $1,324 \pm 0,103$ \\
Diámetro $(\mathrm{cm})$ & $1,425 \pm 0,069$ \\
Fruto $(\mathrm{g})$ & $1,797 \pm 0,266$ \\
Pulpa (exocarpo y mesocarpo) $(\mathrm{g})$ & $0,412 \pm 0,081$ \\
Semilla $(\mathrm{g})$ & $1,385 \pm 0,215$ \\
Pulpa $(\%)$ & $22,966 \pm 3,227$ \\
\hline
\end{tabular}

${ }^{k}$ Promedio \pm desviación estándar 


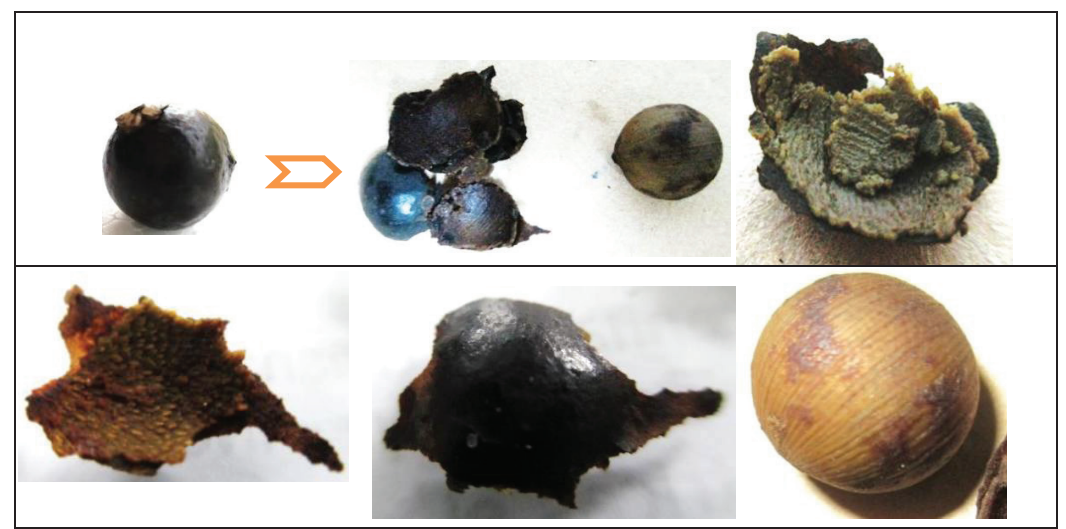

Figura 2. Fruto de Euterpe precatoria Mart. se observa exocarpo, mesocarpo y semilla

El despulpado de Euterpe precatoria presentó dificultades debido a la poca proporción de pulpa en la fruta $(22,97 \%)$ y la poca hidratación lo que dificultó la separación del exospermo y endospermo de las semillas. La pulpa obtenida se congeló a- $10^{\circ} \mathrm{C}$ y se almacenó para su uso. En la tabla 2 se observa el rendimiento de despulpado. Para la aplicación de los métodos de extracción, fue necesario descongelar y secar la muestra; esto se realizó en estufa de aire circulante a $45^{\circ} \mathrm{C}$ por 18 horas.

Tabla 2. Evaluación de rendimiento en la obtención de pulpa de E. precatoria Mart.

\begin{tabular}{ll}
\hline Fruta fresca & $100 \mathrm{~kg}$ \\
Pulpa congelada $\left(-10^{\circ} \mathrm{C}\right)$ & $20 \mathrm{~kg}$ \\
Pulpa seca $(8 \%$ humedad $)$ & $10,225 \mathrm{~kg}$ \\
Rendimiento Fruta fresca/Pulpa seca\} & $10,375 \%$ \\
Rendimiento Pulpa teórico/Pulpa seca\} & $45,1 \%$ \\
\hline
\end{tabular}

El análisis químico proximal de la pulpa seca (tabla 3), que fue utilizada directamente para la extracción de aceite, fue realizado por triplicado. El contenido de cenizas, referida a la presencia de sales minerales, fue similar a lo reportado para la especie de Euterpe olaraceae Mart. por Rojano ${ }^{19}$ y Rufino ${ }^{20}$ (2-7\%). Además, coincide por lo reportado por Castillo (2012) $)^{12}$ que señala valores de porcentaje de ceniza de 1,5 a 4\% para E. precatoria. El contenido de lípidos totales fue superior a lo descrito por Castillo12 que reporta un contenido de lípidos de $11,5 \%$ en pulpa sin tratamiento, pero menor al $37 \%$ reportado por Peña et al ${ }^{14}$ y el $49,4 \%$ obtenido para E. oleracea por Sanabria ${ }^{21}$. Es importante tener en cuenta que los lípidos del fruto se pierden en el proceso de acondicionado (selección y lavado). En esta investigación se analizó la pulpa acondicionada para la extracción de aceite, la cual pasó por un riguroso proceso de selección, lavado y desinfección. Por otro lado, el contenido de proteína hallado sobrepasó lo mencionado por Castillo12, aunque la pulpa de esta especie no puede ser considerada como un alimento fuente de proteínas. El contenido de carbohidratos fue similar a lo reportado por Sanabria ${ }^{21}$, para la especie E.olaraceae, pero inferior a lo reportado por Castillo $^{12}$. 
Tabla 3. Composición proximal de la pulpa de E. precatoria Mart.

\begin{tabular}{lll}
\hline Componentes & Base seca $(\mathrm{BS})^{*}$ & Base húmeda(BH) \\
Humedad & $8,5334 \pm 0,005$ & 7,805 \\
Lípidos totales & $27,2809 \pm 0,01$ & 24,952 \\
Proteínas & $2,3567 \pm 0,1010$ & 2,155 \\
Fibras & $0,3220 \pm 0,0006$ & 0,295 \\
Carbohidratos & $56,6990 \pm 0,099$ & 51,863 \\
Cenizas & $5,1301 \pm 0,00095$ & 4,692 \\
\hline
\end{tabular}

* Promedio \pm desviación estándar $(\overline{\mathrm{X}} \pm S)$

El rendimiento de los métodos de extracción, aplicados para la obtención de aceite de los frutos de Euterpe precatoria Mart., se calculó en función del peso de aceite obtenido y del contenido de aceite en la muestra deducido en base al porcentaje de lípidos, hallado en el análisis químico proximal como recomienda Gomes ${ }^{22}$. El rendimiento de extracción por prensado en frío fue de $31,9 \%$, con la ventaja de obtener aceite de manera directa; es decir, sin necesidad de un tratamiento posterior para su separación; además, se buscó preservar los compuestos antioxidantes del aceite y minimizar el impacto medioambiental en comparación con los métodos convencionales de extracción. Respecto a la extracción con pre tratamiento enzimático-prensado en frío, el rendimiento fue $35,3 \%$; este proceso permitió evaluar el efecto de la incorporación de enzimas sobre el rendimiento de extracción de aceite por prensado en frío. La extracción por prensado con el uso de enzimas es la operación extractiva menos estudiada, a pesar de que presenta ventajas medioambientales frente al uso de hexano y de productividad frente a la extracción acuosa; el rendimiento de extracción del aceite fue $35,28 \%$. Sin embargo, no hay diferencia de rendimiento estadísticamente significativa al comparar ambos métodos de extracción.

El método con mayor rendimiento de extracción fue el de extracción con solventes $(42,6 \%)$; esto debido a la afinidad de los lípidos por los solventes utilizados 2-propanol: etanol: hexano (1:2:1) y el tiempo de contacto ( 8 horas). Sin embargo, la capacidad de la pulpa de absorber solvente al inicio del proceso es un punto crítico ya que significa la inversión de mayor cantidad de solventes, haciendo el proceso costoso; además de la necesidad de incluir una operación de separación en el proceso para eliminar y evitar solventes residuales en el aceite. Este resultado está próximo a lo reportado por Ligia et al..$^{23}$, que mencionan valores de porcentaje de recuperación del aceite de 30-48\%; este método presentó una diferencia de rendimiento estadísticamente significativa al compararlo con los métodos de prensado y pre tratamiento enzimático-prensado.

Como se observa en la tabla 4, la refracción y densidad relativa de los aceites obtenidos por los métodos de prensado, pre tratamiento enzimático y solventes, no presentaron diferencias significativas entre los valores reportados; estos estuvieron dentro de rango de los valores indicados para aceites cosméticos de asaí 0,800-0,950 e índice de refracción 1,35-1,4724. 
El índice de acidez de los aceites obtenidos fue alto, lo que indica que desde el proceso de obtención de pulpa y extracción del aceite se inició el proceso hidrolítico. Por otro lado, el índice de acidez puede incrementarse en el caso de aceites extraídos por prensado por la presencia de pigmentos y compuestos antioxidantes, como clorofila y carotenoides que contiene el aceite. En el caso de aceite extraído por solventes el índice es mucho mayor, presentando una diferencia estadísticamente significativa con los valores reportados por los aceites por prensado y pre tratamiento enzimático-prensado, esto debido al mayor tiempo de extracción y a los procesos de separación del solvente y el aceite posterior a la obtención del extracto, además del contacto prolongado y la aplicación de temperatura $\left(40^{\circ} \mathrm{C}\right)$ en el proceso. Los valores de acidez antes descritos son aceptables para los aceites de uso cosmético, tomando como referencia la ficha técnica de aceite de asaí de la especie Euterpe olaraceae, que indica un índice de acidez máximo de $10 \mathrm{mg} \mathrm{KOH} / \mathrm{g}^{24}$. El índice de peróxido para los aceites obtenidos por los métodos en estudio fueron menores a 3,1 meq $\mathrm{O}_{2} / \mathrm{kg}$ de aceite, aunque para aceites con fines alimenticios el valor es alto, en el caso de aceites de uso cosmético el valor máximo aceptado es de $5 \mathrm{meq} \mathrm{O}_{2} / \mathrm{kg}$ de aceite24. Sin embargo, más allá de los atributos de calidad de los aceites obtenidos a través del prensado y el método de pre tratamiento enzimático-prensado, el contenido de ácido graso libre y color fueron menos intensos que del aceite extraído por solventes. Los valores de yodo y saponificación se encontraron dentro de rango de lo indicado para el aceite cosmético de asaí que indica valores de índice de yodo de 118-145g I2/100g y valores máximos para el índice de saponificación de $200 \mathrm{mg}$ de $\mathrm{KOH} / \mathrm{g}^{24}$.

Tabla 4. Características fisicoquímicas de los aceites obtenidos por los métodos de extracción en estudio.*

\begin{tabular}{llll}
\hline Parámetros & prensado & enzimático & solvente \\
Índice de refracción a $20^{\circ} \mathrm{C}$ & $1,4689 \pm 0,0002$ & $1,4687 \pm 0,0006$ & $1,4660 \pm 0,001$ \\
Densidad relativa a $22^{\circ} \mathrm{C}$ & $0,915 \pm 0,0001$ & $0,916 \pm 0,0002$ & $0,906 \pm 0,0004$ \\
Índice de acidez $(\mathrm{mg} \mathrm{de} \mathrm{KOH} / \mathrm{g})$ & $5,14 \pm 0,29$ & $4,91 \pm 0,34$ & $9,08 \pm 0,62$ \\
Índice de peróxidos $\left(\mathrm{meq} \mathrm{O}_{2} / \mathrm{Kg}\right)$ & $2,08 \pm 0,09$ & $3,16 \pm 0,02$ & $4,25 \pm 0,01$ \\
Índice de saponificación $(\mathrm{mg}$ de $\mathrm{KOH} / \mathrm{g})$ & $265,39 \pm 0,02$ & $285,46 \pm 0,09$ & $250,49 \pm 0,16$ \\
Índice de yodo $(\mathrm{g}$ yodo $/ 100 \mathrm{~g}$ método de & $133,561 \pm 0,003$ & $138,523 \pm 0,021$ & $136,563 \pm 0,017$ \\
Wijs) & & & \\
\hline
\end{tabular}

*Las evaluaciones se realizaron por triplicado \pm desviación estándar.

La capacidad de inhibición de radicales libres de los extractos fue dependiente de la concentración (tabla 5); es importante señalar que las concentraciones utilizadas para los ensayos de DPPH y fenoles totales en el extracto de prensado y pre tratamiento enzimáticoprensado fueron 10 veces más que la concentración utilizada para el de solventes; aunque los extractos en todos los casos fueron obtenidos por una mezcla metanol: agua (8:2), se observó diferencias en la intensidad de color de los mismos y a nivel de IC 50 se reportó diferencias entre los resultados de los aceites obtenidos por cada método de extracción; el menor valor $(6,97 \mathrm{mg} / \mathrm{mL})$ lo mostró el aceite obtenido por prensado debido, probablemente, 
a la extracción de compuestos fenólicos polares; sigue el aceite obtenido con pre tratamiento enzimático-prensado $(3,61 \% \mathrm{mg} / \mathrm{mL})$, donde el pre tratamiento enzimático previo contribuyó al incremento de extracción de compuestos antioxidantes, lo que se evidenció en la reducción de IC 50 a la mitad del valor mostrado para el aceite obtenido por prensado. Se observó el mejor resultado en el aceite obtenido por extracción por solventes que reportó un IC 50 de $1,82 \mathrm{mg} / \mathrm{mL}$; esta variación puede deberse a la afinidad de los componentes antioxidantes al solvente utilizado en la extracción. En relación a ello, Rufino ${ }^{20}$ ha reportado en el estudio de aceite de asaí obtenido de la variedad Euterpe oleraceae-BRS Pará, donde sugiere que la mayor capacidad antioxidante en el aceite de asaí lo presentan los antioxidantes de naturaleza apolar y que la separación de esta fracción puede ser un modo de concentrar los extractos antioxidantes del aceite. Comparando los resultados de esta investigación, en relación al IC 50 de extractos de aceites de E. precatoria con los reportados por Kang et al. ${ }^{11} \quad(0,167 \mathrm{mg} / \mathrm{mL})$ para extractos de pulpa de E. precatoria, se observa que el valor es superior a lo reportado en esta investigación, debido probablemente a la reducción de la concentración de compuestos antioxidantes que logran transferirse a los aceites al momento de su extracción. Sin embargo, ninguno de los extractos mostró mayor actividad que el Trolox utilizado como estándar, que reportó un IC 50 de $0,001 \mathrm{mg} / \mathrm{mL}$.

Tabla 5. Capacidad de inhibición de radicales libres en los aceites obtenido por los diferentes métodos de extracción

\begin{tabular}{|c|c|c|c|c|c|}
\hline & $\begin{array}{c}\text { conc } \\
(\mathrm{mg} / \mathrm{ml})\end{array}$ & promedio & $\begin{array}{l}\text { Desviación } \\
\text { estándar }\end{array}$ & $\begin{array}{c}\text { \% Inhibición de radicales } \\
\text { libres }\end{array}$ & IC $50 \mathrm{mg} / \mathrm{mL}$ \\
\hline \multirow{4}{*}{ 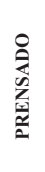 } & 4 & 0,404 & 0,001 & 45,61 & \multirow{4}{*}{6,97} \\
\hline & 8 & 0,373 & 0,001 & 52,85 & \\
\hline & 16 & 0,340 & 0,047 & 61,57 & \\
\hline & 32 & 0,311 & 0,005 & 66,30 & \\
\hline \multirow{4}{*}{ 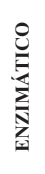 } & 4 & 0,322 & 0,001 & 53,29 & \multirow{4}{*}{3,61} \\
\hline & 8 & 0,287 & 0,001 & 59,29 & \\
\hline & 16 & 0,244 & 0,004 & 69,21 & \\
\hline & 32 & 0,158 & 0,003 & 77,39 & \\
\hline \multirow{4}{*}{ 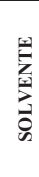 } & 0,4 & 0,332 & 0,001 & 53,76 & \multirow{4}{*}{1,82} \\
\hline & 0,8 & 0,250 & 0,001 & 64,13 & \\
\hline & 1,6 & 0,200 & 0,001 & 76,59 & \\
\hline & 3,2 & 0,116 & 0,001 & 100,00 & \\
\hline \multicolumn{5}{|c|}{ Estándar (Trolox) } & 0,001 \\
\hline
\end{tabular}

El contenido de fenoles totales de los aceites obtenidos por los diferentes métodos de extracción (tabla 6) fue superior a los resultados reportados por Gordon et al. ${ }^{26}$, que reportó valores de 73,0 \pm 4,8 y 31,2 $\pm 2,6 \mathrm{mg}$ ácido gálico/g de peso seco de extracto de las variedades E.precatoria y E.oleracea, observándose una mayor presencia de compuestos fenólicos en la especie en estudio. Confirmando lo reportado por Kang et al. ${ }^{11}$, respecto al predominio de compuestos fenólicos en E. precatoria, en comparación a E. oleraceae. 
Tabla 6. Compuestos fenólicos en aceite expresados en equivalencia de ppm de ácido gálico (AG)

\begin{tabular}{ccccc}
\hline & $\mathbf{m g} / \mathbf{m l}$ & absorbancia promedio & ppm AG & $\begin{array}{c}\text { mg AG/100g de } \\
\text { aceite }\end{array}$ \\
Prensado & 32 & $0,348 \pm 0,001$ & 336,554 & 1050 \\
Enzima & 32 & $0,127 \pm 0,002$ & 122,383 & 381,25 \\
Solvente & 3,2 & $0,365 \pm 0,001$ & 352,335 & 11010,5 \\
\hline
\end{tabular}

\section{CONCLUSIONES}

- El método de extracción con mayor rendimiento fue con solventes $(42,58 \pm 1,76)$. Entre los rendimientos de extracción de los métodos de prensado y pretratamiento enzimáticoprensado, no hubo diferencia estadísticamente significativa de los rendimientos de extracción.

- Las características fisicoquímicas del aceite son similares en las muestras obtenidas por prensado y extracción con pretratamiento enzimático-prensado, siendo estadísticamente significativa la diferencia entre éstos y el aceite obtenido por extracción por solventes el cual reportó una menor calidad.

- La capacidad antioxidante más alta $(\mathrm{IC} 50=1,82 \mathrm{mg} / \mathrm{mL})$ y la mayor proporción de compuestos fenólicos totales $(11010,5 \mathrm{mg} \mathrm{AG} / 100 \mathrm{~g})$ la reportó el aceite extraído por solventes.

\section{AGRADECIMIENTO}

Se agradece a la Dra. Arilmí Gorriti G. por el apoyo incondicional en calidad de asesora en el desarrollo de esta investigación como parte de la tesis para obtener el grado académico de Magister en Productos Naturales y Biocomercio - UNMSM

\section{BIBLIOGRAFÍA}

1. Balick MJ. Amazonian oil palms of promise: a survey. Econ Bot. 1979;33(1):11-28.

2. Balslev H, Grandez C, Zambrana NYP, Møller AL, Hansen SL, Biología RP De, et al. Palmas (Arecaceae) útiles en los alrededores de Iquitos, Amazonía Peruana. Rev Perú Biol. 2008;15 (Supl.1:121-32.

3. Zambrana NYP, Byg A, Svenning J-C, Moraes M, Grandez C, Balslev H. Diversity of palm uses in the western Amazon. Biodivers Conserv [Internet]. 2007 [cited 2013 Dec 13];16(10):2771-87. Available from: http://link.springer.com/article/10.1007/s10531007-9218-y

4. Serviço Florestal Brasileiro. Açaí - Euterpe precatoria Guia da Espéciee [Internet]. Brasília - DF; 2001. Available from: http://www.florestal.gov.br/guia-de-especies-pfnm/ view-category

5. Kahn F. Palms as key swamp forest resources in Amazonia. For Ecol Manage [Internet]. 1991 [cited 2013 Dec 11];38(3):133-42.

6. Daisy A. Pereira Gomes-Silva. Palm trees and diverse other species [Internet]. 2010 p. 191-6. Available from: www.fao.org/docrep/015/i2360e/i2360e04.pdf 
7. Mesa L, Galeano G. Usos de las palmas en la amazonia Colombiana. 2013;35(2):35169.

8. Escriche I, Restrepo J. Composition and nutritive value of Amazonian palm fruits. Food Nutr [Internet].1999[cited 2014 Nov 10]; Available from:

http://www.ingentaconnect.com/content/nsinf/fnb/1999/00000020/00000003/art00014

9. Wadt LH de O, Rigamonte - Azevedo OC, Linhares Ferreyra EJL, Brasil da Cunha Cartaxo CB da C. Manejo de açaí solteiro (Euterpe precatoria Mart.) para produção de frutos. 2007.

10. Sosnowska J, Balslev H. Redalyc.American palms used for medicine, in the ethnobotanical and pharmacological publications. Rev Peru Biol. 2008;15 (supl.1:143-6.

11. Kang J, Thakali KM, Xie C, Kondo M, Tong Y, Ou B, et al. Bioactivities of açaí (Euterpe precatoria Mart.) fruit pulp, superior antioxidant and anti-inflammatory properties to Euterpe oleracea Mart. Food Chem [Internet]. 2012 Aug [cited 2014 Nov 10];133(3):671-7. Available from: http:/www.sciencedirect.com/science/article/pii/ S0308814612000817

12. Castillo Q. YM, Lares A. M, Hernandez G. Ms. Caracterización Bromatológica y Fisicoquímica del Fruto Amazónico Asaí (Euterpe Precatoria Mart). Vitae [Internet]. Enero-Abril 2012 [cited 2014Nov10];19(1):309-11.Available from: http://www.redalyc. org/articulo.oa?id=169823914095

13. Pacheco-Palencia L a., Duncan CE, Talcott ST. Phytochemical composition and thermal stability of two commercial açai species, Euterpe oleracea and Euterpe precatoria. Food Chem [Internet]. Elsevier Ltd; 2009 Aug [cited 2012 Nov 7];115(4):1199-205. Available from: http://linkinghub.elsevier.com/retrieve/pii/S0308814609000715

14. Peña L, Barrera G J, Hernández G. M. Obtención de Pulpa de Asai (Euterpe Precatoria Mart.) en la amazonía norte colombiana. Vitae. 2012;19(1):147-9.

15. AOAC. Official Methods of Analysis. 19 th. Dr. George W. Latimer J, editor. 2012.

16. Parry, J., L. SU, M. Luther, K. Zhou, M. P. Yurawecz, P. Whittaker, and L. YU.. Fatty acid composition and antioxidant properties of cold-pressed marionberry, boysenberry, red raspberry, and blueberry seed oils. J Agr Food Chem 2005; 53: 566-573

17. Brand-William, W., Cuvelier, M. E., \&Berset, C..Use of a free radical method to evaluate antioxidant activity.LebensmWissTechnol1995; 28: 25-30.

18. Chaovanalikit, A., Wrolstad, R. E. Total anthocyanins and total phenolics of fresh and processed cherries and their antioxidant properties J Food Scien2004., 69 (1): 67-72.

19. Rojano, B.A.; Vahos, I.C.Z.; Arbeláez, A.F.A.; Martínez, A.J.M.; Correa, F.B.C.; Carvajal, L.G. Polifenoles y actividad antioxidante del fruto liofilizado de palma naidi (açai colombiano) (Euterpe oleracea Mart). Rev.Fac.Nal.Agr.Medellín. 2011, 64: 62136220.

20. Rufino M, Pérez-Jiménez J. Açaí ( Euterpe oleraceae)'BRS Pará': A tropical fruit source of antioxidant dietary fiber and high antioxidant capacity oil. Food Res[Internet]. 2011 [cited 2014 Nov 10];44:2100-6. Available from: http://www.sciencedirect.com/science/article/pii/S0963996910003194

21. Sanabria N, Sangronis E. Caracterización del acai o manaca ( Euterpe oleracea Mart .): un fruto del Amazonas. Arch Latinoam Nutr. 2007;57(1):94-8. 
22. Gomes De Brito Mariano R, Couri S, Freitas Pereira S. Enzymatic technology to improve oil extraction from Caryocar brasiliense camb. (Pequi) Pulp. Rev Bras Frutic [Internet]. 2009 Sep;31(3):637-43.Available from:http://www.scielo.br/scielo.php?script=sci_ arttext\&pid=S0100-29452009000300003\&lng=en\&nrm=iso\&tlng=en

23. Ligia I, Rodriguez L, Hasbleidy Ramirez. Potencial de recuperación de aceite a partir de pulpa de asaí ( Euterpe oleracea Mart . ) producido en el pacífico colombiano. Bogotá, Colombia; 2012 p. 40-53.

24. Natural Sourcing LLC. INCI: Acai (Euterpe oleracea) Pulp Oil [Internet]. Acai Berry Oil. 2013 [cited 2014 Nov 10]. Available from: http://www.pdfio.net/k-5036486.html

25. Gonçalves GMS, Santos NP, Srebernich SM. Atividades antioxidante e antimicrobiana dos extratos de própolis e açaí (Euterpe oleracea Mart). Rev Ciencias Farm Basica e Apl [Internet]. 2011;32(3):349-56. Available from: http://www.scopus.com/inward/record. url?eid=2-s2.0-84860860300\&partnerID=tZOtx3y1

26. Gordon A, Cruz APG, Cabral LMC, de Freitas SC, Taxi CMAD, Donangelo CM, et al. Chemical characterization and evaluation of antioxidant properties of Açaí fruits (Euterpe oleraceae Mart.) during ripening. Food Chem [Internet]. 2012 Jul [cited 2014 Nov 6];133(2):256-63. Available from: http://www.sciencedirect.com/science/article/ pii/S0308814612000179 